\title{
Effect of an iris-fixated intraocular lens on corneal metabolism: a numerical study
}

Peyman Davvalo Khongar ${ }^{1}$, Jan Oscar Pralits ${ }^{1}$, Xi Cheng ${ }^{2}$, Peter Pinsky ${ }^{2}$, Paolo Soleri ${ }^{3}$, Rodolfo Repetto ${ }^{1}$

${ }^{1}$ Department of Civil, Chemical and Environmental Engineering, University of Genoa, Genoa, Italy; 'Department of Mechanical Engineering, Stanford University, Stanford, CA, USA; ${ }^{3}$ Ophtec BV, Groningen, Netherlands

\section{Abstract}

One of the possible risks associated with the implant of iris-fixated phakic intraocular lenses ( $\mathrm{plOL}$ ) is loss of corneal endothelial cells. We hypothesize that this might be due to alterations in corneal metabolism secondary to the lens implantation. To verify the feasibility of this assumption, we propose a mathematical model of the transport and diffusion of metabolic species in the anterior chamber and the cornea, coupled to a model of aqueous flow. Results are obtained both with and without the $\mathrm{pIOL}$ in the case of closed eyelids. The results suggest that glucose availability may be significantly reduced at the corneal endothelium. However, it must still be verified whether this finding has clinical relevance.

Keywords: aqueous flow, corneal metabolism, intraocular lens

\section{Introduction}

In recent years, pIOL have gained widespread acceptance for the correction of visual acuity deficiencies, such as myopia. These lenses are implanted surgically into the anterior chamber of the eye and fixated to the iris by special claws. The procedure is considered very safe; however, a possible complication is the loss of corneal endothelial cells. We hypothesize that this might be due to alterations in

Correspondence: Peyman Davvalo Khongar, Department of Civil, Chemical and Environmental Engineering, University of Genoa, Via Montallegro 1, 16145, Genova, Italy.

E-mail: peyman.davvalo.khongar@edu.unige.it 
corneal metabolism after pIOL implantation.

Although considerable research has been devoted to mathematical modeling of corneal metabolism, less attention has been paid to include the effect of metabolic species transport across the anterior chamber by the aqueous flow. In this study, we investigate corneal metabolism with and without a $\mathrm{pIOL}$, accounting for aqueous flow in the anterior chamber. Since the cornea receives glucose mostly from the aqueous humor, and following the observation that the $\mathrm{PIOL}$ modifies aqueous flow characteristics, ${ }^{1}$ we speculate that $\mathrm{pIOL}$ implantation may affect corneal metabolism.

When the eyelids are open, the thermal flow and the flow generated by eye rotations are likely intense enough so that the concentration of each metabolic species in the anterior chamber can be considered constant, and consequently, the effect of a pIOL in the delivery of metabolic species to the cornea can be neglected. On the other hand, when the eyelids are closed, aqueous flow is only induced by the production/drainage mechanism ${ }^{1}$ and the resulting velocities are very small. Therefore, it is likely that the concentration of metabolic species will be variable across the anterior chamber and affected by the presence of the pIOL. Thus, we focus on the closed eyelid case.

\section{Methods}

In the present work, we adopt a model for oxygen, lactate, and glucose transport, based on that proposed in by Chhabra et al. ${ }^{2}$ and Pinsky, ${ }^{3}$ considering the role of aqueous humor flow in transporting metabolic species. We improve the previous models by using a sigmoidal oxygen consumption model, as proposed in Alvord et $a l^{4}$ We adopt an idealized axisymmetric shape of the anterior chamber and consider three superposed corneal layers (endothelium, stroma, epithelium) based on previous work (Fig. 1). ${ }^{1,3}$ The geometry of the pIOL has been provided by Ophtec BV; Groningen, Netherlands.

Results are obtained by solving the Navier-Stokes equations for the fluid flow and the following advection-diffusion equations in the aqueous for oxygen tension (denoted by a subscript O), glucose (subscript G), and lactate (subscript L) concentrations:

$$
\begin{aligned}
& k_{\mathrm{o}} \frac{\partial p_{\mathrm{o}}}{\partial t}+k_{\mathrm{o}}(\mathrm{u} \cdot \nabla) p_{\mathrm{o}}-D_{\mathrm{o}} k_{\mathrm{o}} \nabla^{2} p_{\mathrm{o}}=0 \\
& \frac{\partial C_{G}}{\partial t}+(\mathrm{u} \cdot \nabla) C_{G}-D_{G} \nabla^{2} C_{G}=0 \\
& \frac{\partial C_{L}}{\partial t}+(\mathrm{u} \cdot \nabla) C_{L}-D_{L} \nabla^{2} C_{L}=0
\end{aligned}
$$

where $p_{O}, c_{G}$, and $c_{L}$ are the oxygen tension, glucose, and lactate ion concentrations, 


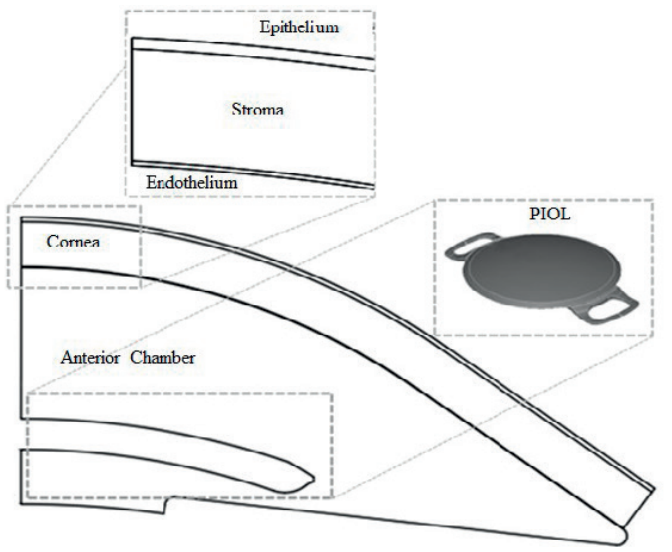

Fig. 1. Cross-section of the idealized anterior chamber with corneal layers and fixated pIOL.

respectively, and $u$ is the flow velocity vector. $D_{i}(i=0, G, L)$ are the diffusion coefficients and $k_{0}$ is Henry's constant.

The transport of metabolic species in the cornea is described by the following equations:

$$
\begin{aligned}
& k_{O} \frac{\partial p_{O}}{\partial t}-D_{O} k_{O} \nabla^{2} p_{O}+Q_{O}=0 \\
& \frac{\partial C_{G}}{\partial t}-D_{G} \nabla^{2} C_{G}+Q_{G}=0 \\
& \frac{\partial C_{L}}{\partial t}-D_{L} \nabla^{2} C_{L}-Q_{L}=0
\end{aligned}
$$

Here, $Q_{L}$ and $Q_{G}$ are the lactate production and glucose consumption rates, respectively, ${ }^{2}$ and $Q_{0}$ is the oxygen consumption rate given by:

$$
Q_{0}=\frac{Q_{Q_{\text {max }}}^{i}}{1+e^{-\frac{\left(P_{0}-P_{\text {ex }}\right)}{R}}} \frac{C_{G}}{C_{G}+K_{G}^{O}}\left(1+0.8 \frac{7.6-p H}{K_{p H}+7.6-p H}\right)
$$

In the above expression $p_{c r t}$ and $R$ are coefficients of the sigmoidal oxygen consumption model. ${ }^{4} \mathrm{~K}_{\mathrm{pH}}$ is set to be 0.1 and $\mathrm{K}_{\mathrm{G}}^{0}$ is the glucose Monod dissociation equilibrium. $Q_{Q_{\max }^{i}}(i=\mathrm{O}, \mathrm{G}, \mathrm{L})$ is the saturation oxygen consumption rate. ${ }^{2}$ For the closed eyelids case, we set $\mathrm{pH}=7.39 .^{5}$

\section{Results}

The results presented below refer to the case of closed eyelids. Steady-state simulations demonstrate that when the eyelids are closed, the presence of a $\mathrm{plOL}$ 


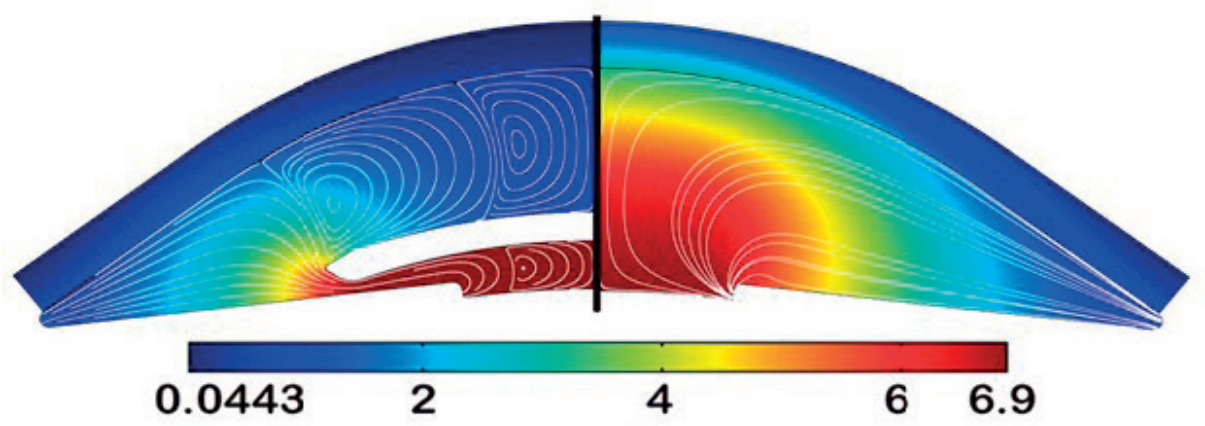

Fig. 2. Glucose concentration shown by colors and flow streamlines in white with (left) and without (right) a pIOL, in the case of closed eyelids.

has a strong influence on glucose availability on the cornea, as shown in Figure 2. Since the aqueous flow is diverted towards the periphery of the anterior chamber, glucose is depleted in a region anterior to the $\mathrm{PIOL}$ in comparison to the case with no pIOL.

Unsteady simulations have also been carried out, starting from conditions representative of open eyelids. As illustrated in Figure 3, glucose concentration at the center of the cornea declines progressively in time and asymptotically reaches the steady state. However, several hours are needed for this steady solution to be reached. During sleep, the typical time interval between successive rapid eye

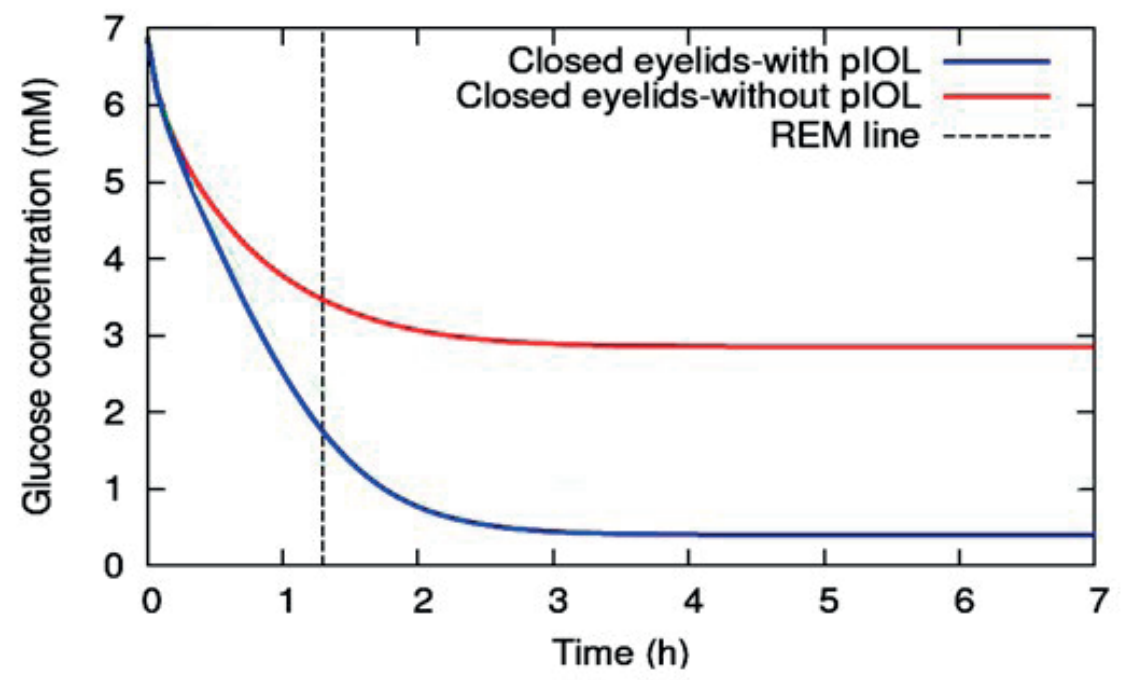

Fig. 3. Time evolution of glucose concentration at the center of cornea with (blue) and without (red) pIOL. The dashed line represents the REM time of $90 \mathrm{~min}$. 
movements (REMs) is 90 minutes. REMs are expected to make the glucose distribution more uniform due to the motion of aqueous humor. It can thus be conceivable that the steady state is unlikely to be ever reached. Nevertheless, the glucose reduction in the time intervals between successive REM phases is significant.

\section{Conclusion}

Our findings demonstrate that in the presence of an iris-fixated pIOL, when the eyelids are closed, glucose concentration at the corneal endothelium may decline significantly. It must be verified whether this has a clinical relevance.

\section{Acknowledgements}

This work was supported financially by Ophtec BV; Groningen, Netherlands.

\section{References}

1. Repetto R, Pralits, JO, Siggers, JH, Soleri P. Phakic iris-fixated intraocular lens placement in the anterior chamber: effects on aqueous flow. Invest Ophthalmol Visual Sci. 2015;56(5):3061-3068.

2. Chhabra M, Prausnitz JM, Radke CJ. Diffusion and monod kinetics to determine in vivo human corneal oxygen-consumption rate during soft contact-lens wear. J Biomed Mater Res B Appl Biomater. 2009;90(1):202-209.

3. Pinsky PM. Three-Dimensional modeling of metabolic species transport in the cornea with a hydrogel intrastromal inlay. Invest Ophthalmol Visual Sci. 2014;55(5):3093-3106.

4. Alvord LA, Hall WJ, Keyes LD, Morgan CF, Winterton LC. Corneal oxygen distribution with contact lens wear. Cornea. 2007;26(6):654-664.

5. Bonanno JA, Polse KA. Corneal acidosis during contact lens wear: effects of hypoxia and CO2. Invest Ophthalmol Visual Sci. 1987;28(9):1514-1520. 